\title{
Raloxifene Induces Autophagy-Dependent Cell Death in Breast Cancer Cells via the Activation of AMP-Activated Protein Kinase
}

\author{
Dong Eun Kim, Yunha Kim ${ }^{1,10}$, Dong-Hyung $\mathrm{Cho}^{2}$, Seong-Yun Jeong ${ }^{1,3}$, Sung-Bae $\mathrm{Kim}^{4}$, Nayoung Suh ${ }^{3,5}$, \\ Jung Shin Lee ${ }^{1,6}$, Eun Kyung Choi ${ }^{1,7}$, Jae-Young Koh ${ }^{8}$, Jung Jin Hwang ${ }^{1,3, *}$, and Choung-Soo Kim ${ }^{1,9, *}$
}

\begin{abstract}
Raloxifene is a selective estrogen receptor modulator (SERM) that binds to the estrogen receptor (ER), and exhibits potent anti-tumor and autophagy-inducing effects in breast cancer cells. However, the mechanism of raloxifeneinduced cell death and autophagy is not well-established. So, we analyzed mechanism underlying death and autophagy induced by raloxifene in MCF-7 breast cancer cells.

Treatment with raloxifene significantly induced death in MCF-7 cells. Raloxifene accumulated GFP-LC3 puncta and increased the level of autophagic marker proteins, such as LC3-II, BECN1, and ATG12-ATG5 conjugates, indicating activated autophagy. Raloxifene also increased autophagic flux indicators, the cleavage of GFP from GFP-LC3 and only red fluorescence-positive puncta in mRFP-GFP-LC3expressing cells. An autophagy inhibitor, 3-methyladenine (3-MA), suppressed the level of LC3-II and blocked the formation of GFP-LC3 puncta. Moreover, siRNA targeting BECN1 markedly reversed cell death and the level of LC3-II increased by raloxifene. Besides, raloxifene-induced cell death was not related to cleavage of caspases-7, -9 , and PARP. These results indicate that raloxifene activates autophagy-dependent cell death but not apoptosis. Interestingly, raloxifene decreased the level of intracellular ade-
\end{abstract}

\footnotetext{
${ }^{1}$ Institute for Innovative Cancer Research, University of Ulsan College of Medicine, Asan Medical Center, Seoul 138-736, Korea, ${ }^{2}$ Graduate School of East-West Medical Science, Kyung Hee University, Yongin 446-701, Korea, ${ }^{3}$ Asan Institute for Life Sciences, ${ }^{4}$ Department of Oncology, University of Ulsan College of Medicine, ${ }^{5}$ Department of Medicine, ${ }^{6}$ Department of Internal Medicine, ${ }^{7}$ Department of Radiation Oncology, University of Ulsan College of Medicine, Asan Medical Center, Seoul 138-736, Korea, ${ }^{8}$ Neural Injury Research Center and Department of Neurology, Asan Medical Center, Seoul, Korea, ${ }^{9}$ Department of Urology, University of Ulsan College of Medicine, Asan Medical Center, Seoul, Korea, ${ }^{10}$ Present address: Center for Neuro-Medicine, Brain Science Institute Korea Institute of Science and Technology, Seoul 110-799, Korea

*Correspondence: jjhwang @amc.seoul.kr (JJH); cskim@amc.seoul.kr (CSK)
}

Received 9 July, 2014; revised 30 October, 2014; accepted 10 November, 2014; published online 24 December, 2014

Keywords: AMPK, ATP, autophagy, breast cancer, raloxifene nosine triphosphate (ATP) and activated the AMPK/ULK1 pathway. However it was not suppressed the AKT/mTOR pathway. Addition of ATP decreased the phosphorylation of AMPK as well as the accumulation of LC3-II, finally attenuating raloxifene-induced cell death.

Our current study demonstrates that raloxifene induces autophagy via the activation of AMPK by sensing decreases in ATP, and that the overactivation of autophagy promotes cell death and thereby mediates the anti-cancer effects of raloxifene in breast cancer cells.

\section{INTRODUCTION}

Macroautophagy (autophagy) is a self-digestion mechanism for degrading damaged organelles and misfolded proteins in the lysosomal compartments. Autophagy starts with the formation of double-membraned vesicles, or autophagosomes, which undergo maturation by fusion with lysosomes in order to create autolysosomes. In autolysosomes, the inner membrane of the autophagosome and its contents are degraded by lysosomal enzymes (Eskelinen, 2008; Maiuri et al., 2007). Under metabolic stress, autophagy maintains a balance between synthesis, degradation, and the subsequent recycling of macromolecules and organelles in order to continue survival. On the other hand, the overactivation of autophagy can promote cell death during persistent stress (Eskelinen, 2008; Levine, 2007; Levine and Kroemer, 2008; Morselli et al., 2009).

The paradox that autophagy plays a role in both survival and death is more complicated in cancer cells. The first specific link between autophagy and cancer was reported in 1999 by Levine et al. They reported that BECN1 acts as a tumor suppressor by inhibiting cell proliferation and tumorigenesis both in vitro and in vivo, and that downregulating autophagy may contribute to the progression of breast and other cancers (Liang et al., 1999). It was also reported that autophagy-dependent cell death is induced by many anti-cancer drugs, such as tamoxifen (Hwang et al., 2010), rapamycin (Takeuchi et al., 2005), arsenic trioxide (Kanzawa et al., 2005), and histone deacetylase (HDAC) inhibitors (Liu et al., 2010). These reports suggested that the overactivation of autophagy is an important death mechanism in tumors, where apoptosis is limited. In contrast, several groups report that inhibiting autophagy facilitates tumor 
regression because autophagy promotes the survival of stressed cancer cells (Hippert et al., 2006). For these reasons, the relationship between autophagy and cancer cannot be summarized simply and requires further investigation.

Previously, we reported that tamoxifen induces autophagydependent cell death in MCF-7 cells via the accumulation of intracellular zinc ions and reactive oxygen species (ROS), which finally leads to lysosomal membrane permeabilization (LMP) (Hwang et al., 2010). Tamoxifen is a selective estrogen receptor modulator (SERMs) that binds to the estrogen receptor (ER) and exhibits selective agonistic or antagonistic effects against target tissue (Fabian and Kimler, 2005). Tamoxifen is the first SERM to be used to treat and prevent ER-positive breast cancer (Fisher et al., 1998). Raloxifene has been used to prevent and treat osteoporosis in 2001, since it has an estrogenic activity in bone (Gizzo et al., 2013). In contrast, since it had and anti-estrogenic activity in breast, U.S. Food and Drug Administration (FDA) approved raloxifene for reduction the risk of invasive breast cancer in postmenopausal women with osteoporosis and in postmenopausal women at high risk for invasive breast cancer in 2007 (Powles, 2011). In breast cancer cells, many studies demonstrated that in vivo and in vitro antitumorigenic effect of raloxifene (Shibata et al., 2010; Taurin et al., 2013). One of the these studies, Taurin et al. (2013) reports that raloxifene decreases tumorigenecity, migration, and invasion in breast cancer cells.

In our current study, we evaluated whether raloxifene induces autophagy-dependent mammalian target of rapamycin (mTOR), AMP-activated protein kinase (AMPK), and autophagy, and is accordingly responsible for the anti-proliferative effects of raloxifene on MCF-7 breast cancer cells.

\section{MATERIALS AND METHODS}

Cell culture and drug treatment

MCF-7 human breast cancer cells expressing green fluorescent protein (GFP)-conjugated microtubule-associated protein 1 light chain 3 (LC3) (GFP-LC3-MCF-7) and red fluorescent protein (mRFP)-GFP tandem fluorescent-tagged LC3 (mRFP-GFP-LC3MCF-7) were established as previously described (Hwang et al., 2010). These cells were pre-treated with various concentrations of raloxifene (Cayman, USA) in RPMl1640 medium containing 10\% charcoal-stripped FBS (Thermo Scientific, Germany), 100 $\mathrm{U} / \mathrm{ml}$ penicillin, and $100 \mu \mathrm{g} / \mathrm{ml}$ streptomycin (Invitrogen, USA). Pan-caspase inhibitor, caspase-9 inhibitor (R\&D Systems, USA), 3-methyladenine (3-MA) (Sigma, USA), siRNA control, and siRNA BECN1 (Bioneer, USA) were applied for the indicated times prior to the addition of raloxifene.

\section{Cell viability assay}

CellTiter 96 AQueous One Solution Cell Proliferation Assay (MTS assay) reagent (Promega, USA) was added to each well containing cells that had been treated with various drugs according to the manufacturer's instructions. Cell viability was determined by measuring absorbance at $490 \mathrm{~nm}$ using a Sunrise microplate reader (TECAN, Switzerland).

\section{Trypan blue exclusion assay}

Cells were stained with $0.1 \%$ trypan blue solution (Invitrogen) for $1 \mathrm{~min}$ and counted using a homocytometer under a light microscope. The percentage and total number of stained dead cells were calculated.
ATP measurement

The CellTiter-Glo Luminescent Assay reagent (Promega) was added to each well according to the manufacturer's instructions. The level of ATP was determined using an EnVision Multilabel Reader (Perkin-Elmer, USA) by measuring the luminescent signal.

\section{Western blot analysis}

Western blot analysis was performed, as previously described (Hwang et al., 2010), using antibodies against BECN1, phosphoAMPK (Thr172), AMPK, phospho-ULK1 (Ser317), phosphoULK1 (Ser757), ULK1, phospho-mTOR (Ser2448), mTOR, phospho-AKT (Ser473), AKT, tubulin (Cell Signaling, USA), ATG5 (Abcam, UK), LC3 (NOVUS Biologicals, USA), caspase-7, caspase-9, PARP (Santa Cruz Biochemicals, USA), and actin (Sigma). Actin or tubulin was used as the loading control.

\section{RNA interference and transfection}

Cells were transfected with $0.17 \mu \mathrm{M}$ BECN1 siRNA (Thermo Scientific) or non-targeting control siRNA (Santa Cruz) for $48 \mathrm{~h}$ using Lipofectamin2000 (Invitrogen) according to the manufacturer's instructions.

\section{Autophagic flux analysis}

mRFP-GFP-LC3-MCF-7 cells were fixed with 4\% paraformaldehyde (PFA, Sigma) and stained with $10 \mu \mathrm{M}$ Hoechst33342 (Sigma) after treatment with raloxifene or rapamycin (Sigma). Images of the cells were obtained from the Operetta High Content Imaging System (Perkin-Elmer) and analyzed using the Harmony Analysis Software (Perkin-Elmer). Cells were detected with green (GFP) or red (mRFP) fluorescence. Autophagosomes are yellow puncta and autolysosomes are only red puncta in merged images. Autophagic flux was determined by increased percent of only red puncta in the merged images.

\section{Statistics}

Data were obtained from $\geq 3$ independent experiments and are presented as the mean \pm standard deviation (SD). Statistical evaluations of the results were performed using one-way ANOVA. Data were considered significant at $p<0.05$.

\section{RESULTS AND DISCUSSION}

Raloxifene inhibits the growth of MCF-7 cells

Raloxifene has in vitro anti-estrogen activities on breast cancer cells, and associated with a decreased incidence of invasive breast cancer. Several studies have demonstrated that raloxifene is effective in other cancers such as prostate cancer and myeloma (Olivier et al., 2006; Rossi et al., 2011). However, their mechanism of anti-cancer effects is not established well. To assess the effects of raloxifene on cell growth, MCF-7 breast cancer cells were treated with the indicated concentrations of raloxifene for $48 \mathrm{~h}$, and cell viability and death were examined using the MTS and trypan blue exclusion assays, respectively. Raloxifene efficiently attenuated cell growth and induced cell death in a concentration-dependent manner (Figs. 1A and 1B). We selected $10 \mu \mathrm{M}$ raloxifene, which killed about $50 \%$ of cell within $48 \mathrm{~h}$, for further analysis. Raloxifene-treated cells had rounded up at $24 \mathrm{~h}$, detached from the dish, and died at $48 \mathrm{~h}$ when observed under a light microscope (Fig. 1C). These data indicate that raloxifene induces death in MCF-7 cells.

Raloxifene activates autophagy in MCF-7 cells

To monitor autophagic vacuoles (AVs), we used MCF-7 cells that 

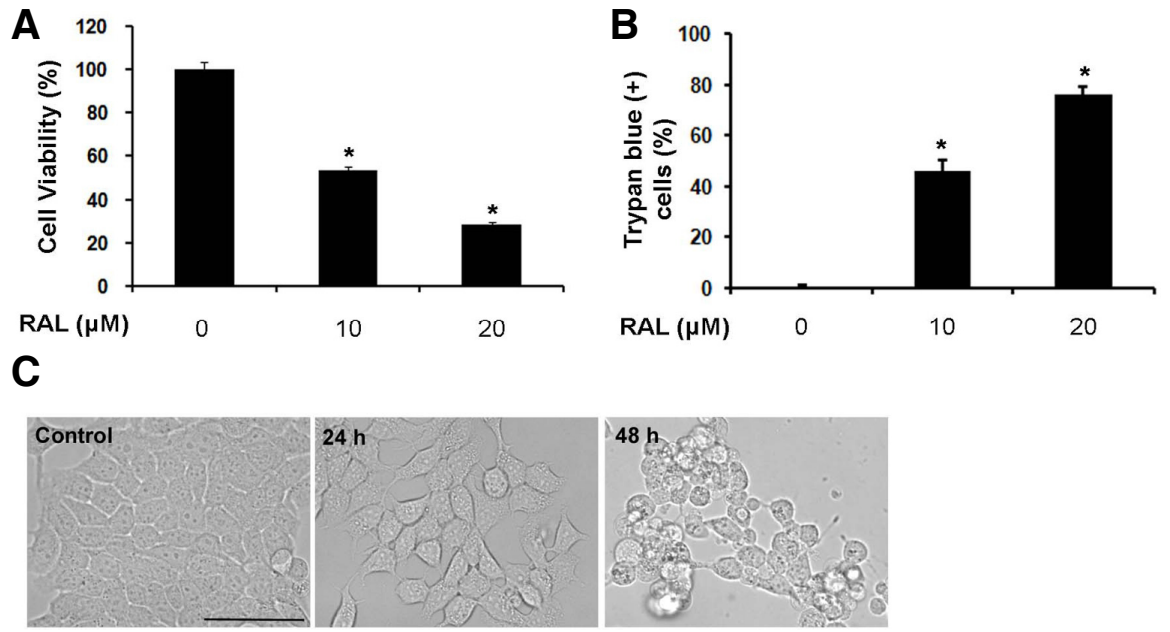

A
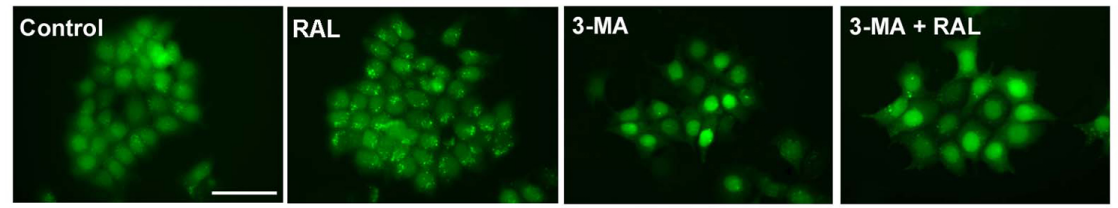

B

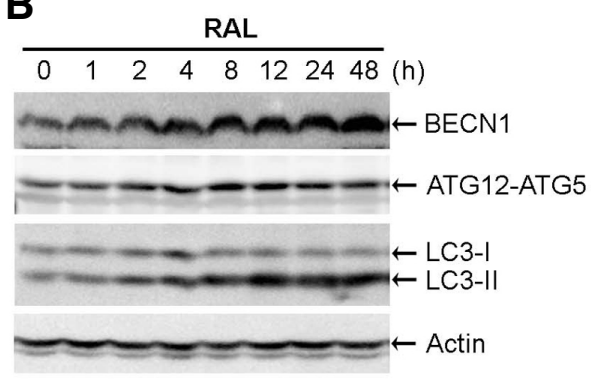

C

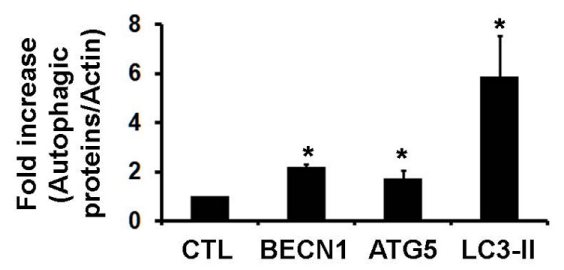

E

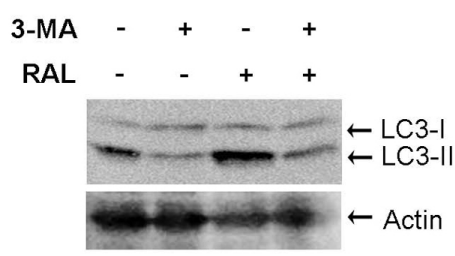

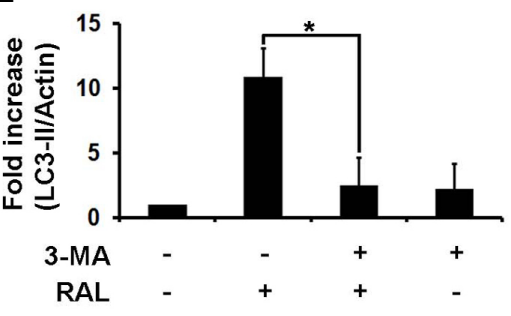

Fig. 1. Raloxifene induces cell death and decreases cell viability in MCF-7 cells. (A) MCF-7 cells were treated with $10 \mu \mathrm{M}$ or $20 \mu \mathrm{M}$ raloxifene (RAL) for $48 \mathrm{~h}$. Cell viability was assessed using the MTS assay (mean $\pm \mathrm{SD} ; \mathrm{n}=$ 3). ${ }^{*} P<0.05$ compared to control. (B) Cell death was evaluated using the trypan blue exclusion assay after treatment with raloxifene for $48 \mathrm{~h}$ (mean $\pm \mathrm{SD} ; \mathrm{n}=3$ ). ${ }^{\star} P<0.05 \mathrm{com}$ pared to control. (C) MCF-7 cells were treated with $10 \mu \mathrm{M}$ raloxifene for 24 or $48 \mathrm{~h}$. Cell morphology was examined using a light microscope (Magnification, 20x; Scale bar, $50 \mu \mathrm{m}$ ).

Fig. 2. Raloxifene induces autophagy in MCF-7 cells. (A) GFP-LC3-MCF-7 cells were pretreated with $4 \mathrm{mM}$ 3-MA for $4 \mathrm{~h}$ and then exposed to $10 \mu \mathrm{M}$ raloxifene for an additional $8 \mathrm{~h}$. These cells were observed under a fluorescent microscope (Magnification, 20x; Scale bar, $50 \mu \mathrm{m}$ ). (B) BECN1, ATG12ATG5, and LC3 were analyzed by Western blot analysis in MCF-7 cells treated with $10 \mu \mathrm{M}$ raloxifene for the indicated times. (C) Bar graph shows the densitometric measurements of autophagic marker proteins expressed in MCF-7 cells treated with $10 \mu \mathrm{M}$ raloxifene for $8 \mathrm{~h}$ (mean $\pm S D ; n=3$ ). Mean intensity was normalized to actin and compared with the each control (mean $\pm \mathrm{SD}, \mathrm{n}=3$ ). ${ }^{*} P<0.05$ compared to control. (D) MCF-7 cells were pretreated with $4 \mathrm{mM}$ 3-MA for $4 \mathrm{~h}$ and then exposed to $10 \mu \mathrm{M}$ raloxifene for an additional $8 \mathrm{~h}$. LC3 was analyzed using Western blot analysis. (E) Bar graph shows the densitometric measurements of the LC3-II in MCF-7 cells which were pretreated with $4 \mathrm{mM}$ 3-MA for $4 \mathrm{~h}$ and then exposed to $10 \mu \mathrm{M}$ raloxifene for an additional $8 \mathrm{~h}$. (mean \pm $\mathrm{SD}, \mathrm{n}=3$ ). ${ }^{*} P<0.05$ compared to raloxifene alone. constitutively expressed GFP-tagged LC3 (GFP-LC3-MCF-7). GFP-LC3 diffuses into the cytoplasm and nucleus under normal conditions, but conjugates with phosphatidylethanolamine (PE) and is incorporated into the AV membrane upon the induction of autophagy. These GFP-positive vacuoles can be visualized using fluorescent microscopy (Dorsey et al., 2009). When we exposed GFP-LC3-MCF-7 cells to raloxifene for $8 \mathrm{~h}$, GFP-positive AVs were obviously apparent in comparison with the sham-washed control cells (Fig. 2A). We also detected autophagic marker proteins using Western blot analysis. Raloxifene augmented the level of BECN1 required for early autophagophore formation, in addition to the ATG12-ATG5 conjugates and LC3-II needed to elongate the autophagosomal membrane in a time-dependent manner (Figs. 2B and $2 \mathrm{C}$ ). Pretreatment with 3-MA, which blocks autophagy by inhibiting class III phosphatidylinositol 3kinase (PI3K), decreased GFP-positive AVs (Fig. 2A) and the level of LC3-II increased following raloxifene treatment (Figs. $2 \mathrm{D}$ and $2 \mathrm{E}$ ), thereby confirming the activation of autophagy by raloxifene.

LC3-II is increased when the production of autophagophores or autophagosomes are increased or the fusion of autophagosomes with lysosomes are inhibited. So it is important to know whether 
A

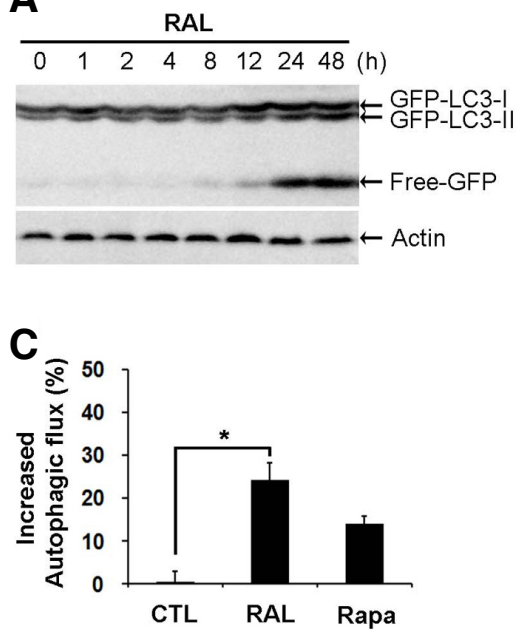

B

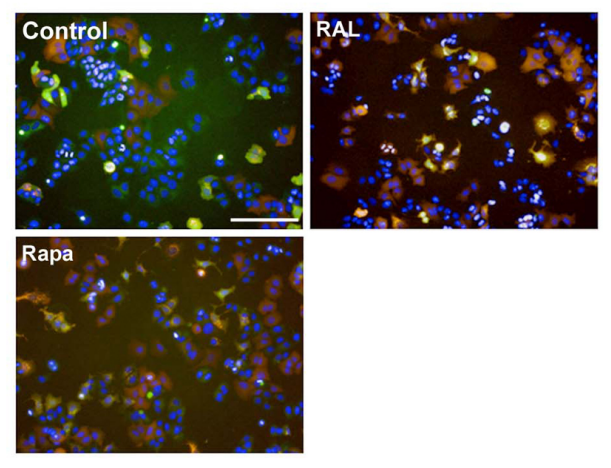

Fig. 3. Raloxifene activates autophagic flux in MCF-7 cells. (A) MCF-7 cells were treated with $10 \mu \mathrm{M}$ raloxifene for the indicated times. GFP was analyzed using Western blot analysis. (B) mRFP-GFPLC3-MCF-7 cells were exposed to 10 $\mu \mathrm{M}$ raloxifene and $400 \mathrm{nM}$ rapamycin (Rapa) for $24 \mathrm{~h}$, and fluorescent images were obtained from the Operetta automated microscope (Magnification, 20x; Scale bar, $50 \mu \mathrm{m})$. The yellow puncta indicate autophagosomes and red puncta represent autolysosomes. Rapamycin was used as a positive control to visualize the autophagic flux. (C) The percent of increased autophagic flux were calculated only red puncta in the merged images. Data are representation of two independent experiments (mean $\pm S D$ ). ${ }^{*} p<0.05$ according to one-way ANOVA. raloxifene activates the whole process of autophagy. This process is called as autophagic flux which includes degradation of the contents of AVs after formation of autolysosome. It was reported that lysosomal hydrolases cleaved GFP-LC3-II and increased free-GFP proteins during the autophagic flux (Balgi et al., 2009). Raloxifene induced a time-dependent increase in free-GFP (Fig. 3A). Besides, we used MCF-7 cells that constitutively expressed mRFP-GFP tandem fluorescent-tagged LC3 (mRFP-GFP-LC3-MCF-7) to monitor autophagic flux. Because GFP fluorescence is unstable in the low $\mathrm{pH}$, it will be quenched in the autolysosomes. But acidic insensitive-mRFP fluorescence will not be quenched (Mizushima et al., 2010). Therefore, while the yellow puncta represent the autophagosomes, the red puncta indicate autolysosomes in the merged fluorescent image, representing autophagic flux. Raloxifene increased the yellow and red puncta (Figs. $3 \mathrm{~B}$ and $3 \mathrm{C}$ ), indicating that raloxifene stimulates autophagic flux as well as the formation of AVs in MCF-7 cells.

Because MCF-7 cells are ER-positive breast cancer cells, we also examined if raloxifene induces autophagy in ER-negative SKBr-3 breast cancer cells and HCT116 colon cancer cells. In accordance with our previous study on tamoxifen (Hwang et al., 2010), raloxifene increased the level of LC3-II in these cell lines (data not shown). These results indicate that either raloxifene or tamoxifen activates autophagy regardless of the ER status in breast cancer and even colon cancer cells.

Raloxifene induces autophagy-dependent cell death in MCF-7 cells

To determine if raloxifene induces autophagy-dependent cell death, cell viability was measured in MCF-7 cells that were treated with raloxifene after BECN1 knockdown using siRNA. RNA interference against $B E C N 1$ recovered the viability of the MCF-7 cells that were treated with raloxifene for $48 \mathrm{~h}$ (Fig. 4A) and reduced the level of LC3-II as well as BECN1 that increased following raloxifene treatment (Fig. 4B). The addition of inhibitors for pan-caspase and caspase- 9 neither reversed the decreased cell viability that occurred following raloxifene treatment (Fig. 4C), nor raloxifene-activated caspase-9 (Fig. 4D). Because MCF-7 cells had Caspase- 3 deleted and expressed functional caspase-7 among various effector caspases, we next examined the cleavage of caspase- 7 and its substrate, PARP.
As expected, raloxifene did not facilitate the cleavage of these proteins (Fig. 4D). These results show that raloxifene induces cell death associated with autophagy, but not apoptosis in MCF-7 cells.

\section{Raloxifene induces autophagy via AMPK activation}

To elucidate the molecular mechanisms that underlie raloxifeneinduced autophagy, we examined the upstream signaling pathways. First, we examined the inhibition of AKT and mTOR, which are well-known mechanisms of autophagy activation (He and Klionsky, 2009; Jung et al., 2010; Ryter et al., 2013; Yang and Klionsky, 2010). In contrast to our expectations, Western blot analysis revealed that the phosphorylation of AKT and mTOR increased following raloxifene treatment. Moreover, raloxifene did not change the phosphorylation of ULK1 at serine 757, an inhibitory site phosphotylated by mTOR (Fig. 5A). These results indicate that raloxifene-activated autophagy is not related to mTOR signaling. We next examined the level of intracellular ATP, because decrease in ATP activates AMPK. Exposure to raloxifene decreased the level of intracellular ATP to $12 \%$ (Fig. 5B), thereby increasing the phosphorylation of threonine 172 on APMK and serine 317 on ULK1 which is required to initiate autophagy (Figs. $5 \mathrm{~A}$ and $5 \mathrm{C}$ ). (Alers et al., 2012; Egan et al., 2011; Kim et al., 2011; Lee et al., 2010). The addition of ATP, which raised the level of intracellular ATP to $36 \%$ (Fig. 5B), rescued the cell viability reduced by raloxifene (Fig. 5D) and decreased phospho-AMPK as well as LC3-II (Figs. 5C). Accordingly, nicotinamide adenine dinucleotide (NAD), which accelerates the production of ATP (Khan et al., 2007), recovered the viability of the raloxifene-exposed cells (Fig. 5D). Collectively, these results suggest that raloxifeneinduced autophagy and death are mediated by the activation of AMPK, without the inhibition of AKT/mTOR pathway.

According to the 1996 study by Bursch et al. (1996) tamoxifen reportedly activates autophagy and induces type II cell death. We have also reported that tamoxifen increases the ROS- and zincmediated overactivation of autophagy, thereby leading to lysosomal membrane permeabilization (LMP) (Hwang et al., 2010). de Medina et al. (2009) reported that tamoxifen and other SERMs activate autophagy by modulating cholesterol metabolism. However, none of these studies described raloxifene in detail. Here, we show that raloxifene increases autophagy- 


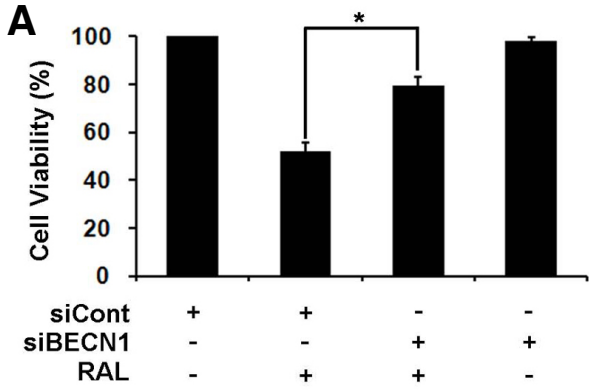

C

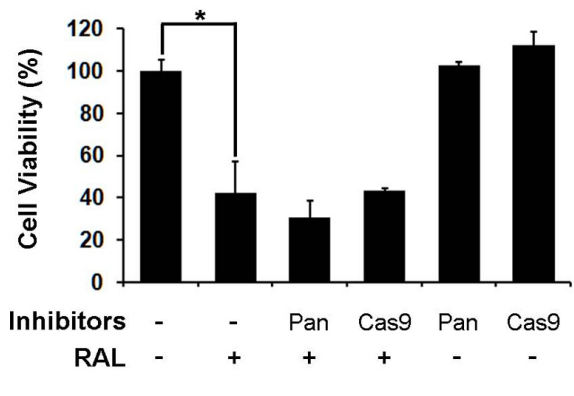

A

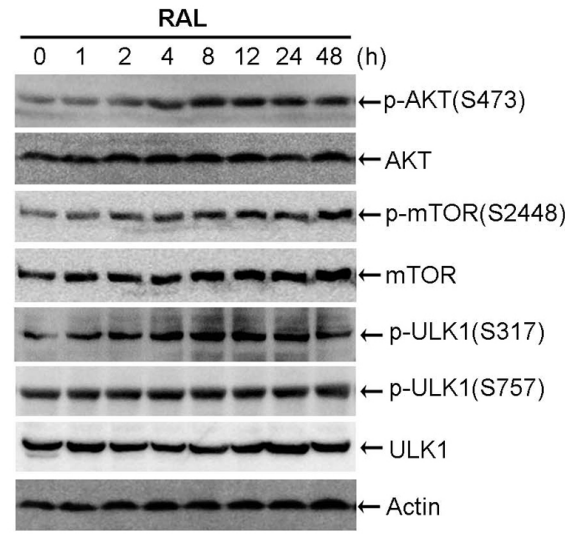

C

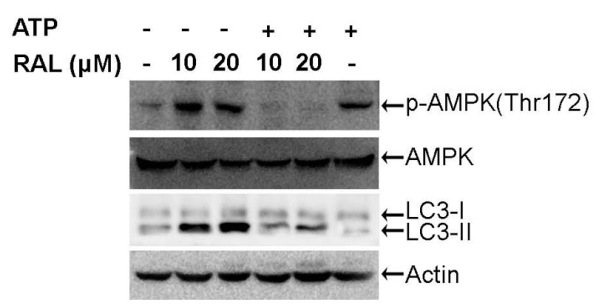

B

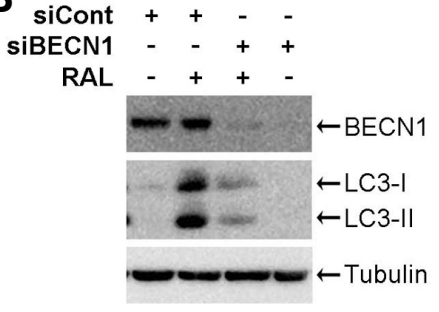

D

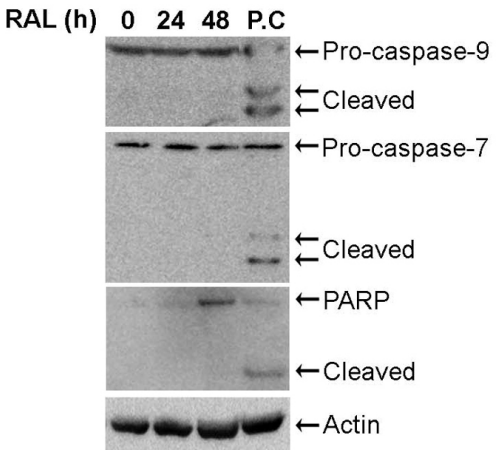

B
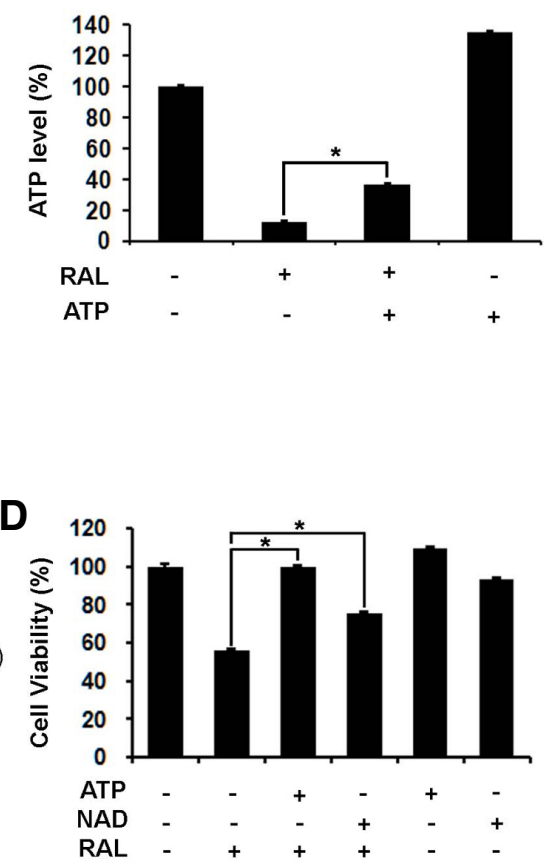

Fig. 4. Raloxifene induces autophagydependent cell death. (A) MCF-7 cells were transfected with $0.17 \mu \mathrm{M}$ nontargeting control siRNA (siCont) or $B E C N 1$ siRNA (siBECN1) for $48 \mathrm{~h}$. Bars denote cell viability of cells treated with $10 \mu \mathrm{M}$ raloxifene for $48 \mathrm{~h}$, and cell viability was assessed using the MTS assay (mean $\pm \mathrm{SD} ; \mathrm{n}=3$ ). ${ }^{*} p<0.05$ according to one-way ANOVA. (B) MCF-7 cells were transfected with $0.17 \mu \mathrm{M}$ siCont or siBECN1 for $48 \mathrm{~h}$. BECN1 and LC3 were analyzed using Western blot analysis. (C) MCF-7 cells were pretreated with $20 \mu \mathrm{M}$ caspase inhibitors for $2 \mathrm{~h}$ and then exposed to $10 \mu \mathrm{M}$ raloxifene for $48 \mathrm{~h}$. Cell viability was measured using the MTS assay (mean $\pm \mathrm{SD} ; \mathrm{n}=3$ ). ${ }^{*} p<0.05$ according to one-way ANOVA. (D) MCF-7 cells were treated with $10 \mu \mathrm{M}$ raloxifene for the indicated times. Caspase-7, -9, and PARP were analyzed using Western blotting. The lysate of the HCT116 cells treated with $10 \mu \mathrm{M}$ PXD101 for $24 \mathrm{~h}$ was used as a positive control (P.C) to assess the cleavage of caspase- $7,-9$, and PARP.

Fig. 5. Raloxifene activates AMPK/ULK1 signaling. (A) MCF-7 cells were exposed to $10 \mu \mathrm{M}$ raloxifene for the indicated times. Phospho-AKT (S473), AKT, phosphomTOR (S2448), mTOR, phospho-ULK1 (S317), phospho-ULK1 (S757), and ULK1 were analyzed using Western blotting. (B) MCF-7 cells were pretreated with 50 $\mu \mathrm{M}$ ATP for $2 \mathrm{~h}$ and then exposed to 10 $\mu \mathrm{M}$ raloxifene for $4 \mathrm{~h}$. Bars denoted the level of ATP measured by the CellTiterGlo Luminescent assay (mean $\pm \mathrm{SD} ; \mathrm{n}=$ 3). ${ }^{*} p<0.05$ according to one-way ANOVA. (C) MCF-7 cells were pretreated with $50 \mu \mathrm{M}$ ATP for $2 \mathrm{~h}$ and then exposed to 10 or $20 \mu \mathrm{M}$ raloxifene for $4 \mathrm{~h}$. The level of phospho-AMPK (Thr172), AMPK, and LC3 were analyzed by Western blotting. (D) MCF-7 cells were pretreated with $50 \mu \mathrm{M}$ ATP or $40 \mu \mathrm{M}$ NAD for $2 \mathrm{~h}$ and then exposed to $10 \mu \mathrm{M}$ raloxifene for $48 \mathrm{~h}$. The cell viability was measured using the MTS assay (mean $\pm S D ; n=3$ ). ${ }^{*} p<0.05$ according to one-way ANOVA. mediated cell death by activating the AMPK pathway via decreases in intracellular ATP in MCF-7 breast cancer cells. The addition of ATP increased the intracellular level of ATP in this experiment, thereby protecting cells from raloxifene-induced cell death. However, we cannot rule out the possibility that extracellular role of ATP. Extracellular ATP binds to specific plasma membrane receptors (called $\mathrm{P} 2$ receptors) and initiates cellular signaling events such as growth, proliferation, and apoptosis (Deli and 
Csernoch, 2008). The anti-cancer activity of ATP has been reported by many groups, which have reported that ATP-activated $\mathrm{P} 2$ receptors decrease tumor growth and increase apoptosis in diverse types of cancers (Hopfner et al., 1998; Wang et al., 2004; White and Burnstock, 2006). Conversely, extracellular ATP activates $\mathrm{P} 2$ receptors followed by increases intracellular calcium and cell proliferation of MCF-7 cells, which is supported by ATP acting as a promoter of cell proliferation and growth (Dixon et al., 1997; Wagstaff et al., 2000). Therefore, further studies on the mechanisms by which raloxifene inhibits $\mathrm{P} 2$ receptor-mediated signaling are needed.

In this study, we suggest that raloxifene-induced decrease in ATP activates AMPK, leading to autophagy and autophagic flux. The overactivation of autophagy can lead to cell death, which can be one of the mechanisms of anti-cancer effect of raloxifene.

\section{ACKNOWLEDGMENTS}

This study was supported by a grant of the Korea Health Technology R\&D Project, Ministry of Health \& Welfare, Republic of Korea (HI06C0868, HI10C2014, and HI09C1345).

\section{REFERENCES}

Alers, S., Loffler, A.S., Wesselborg, S., and Stork, B. (2012). Role of AMPK-mTOR-Ulk $1 / 2$ in the regulation of autophagy: cross talk, shortcuts, and feedbacks. Mol. Cell. Biol. 32, 2-11.

Balgi, A.D., Fonseca, B.D., Donohue, E., Tsang, T.C., Lajoie, P., Proud, C.G., Nabi, I.R., and Roberge, M. (2009). Screen for chemical modulators of autophagy reveals novel therapeutic inhibitors of mTORC1 signaling. PLoS One 4, e7124.

Bursch, W., Ellinger, A., Kienzl, H., Torok, L., Pandey, S., Sikorska, M., Walker, R., and Hermann, R.S. (1996). Active cell death induced by the anti-estrogens tamoxifen and $\mathrm{ICl} 164384$ in human mammary carcinoma cells (MCF-7) in culture: the role of autophagy. Carcinogenesis 17, 1595-1607.

de Medina, P., Payre, B., Boubekeur, N., Bertrand-Michel, J., Terce, F., Silvente-Poirot, S., and Poirot, M. (2009). Ligands of the antiestrogen-binding site induce active cell death and autophagy in human breast cancer cells through the modulation of cholesterol metabolism. Cell Death Differ. 16, 1372-1384.

Deli, T., and Csernoch, L. (2008). Extracellular ATP and cancer: an overview with special reference to P2 purinergic receptors. $\mathrm{Pa}$ thol. Oncol. Res. 14, 219-231.

Dixon, C.J., Bowler, W.B., Fleetwood, P., Ginty, A.F., Gallagher, J.A., and Carron, J.A. (1997). Extracellular nucleotides stimulate proliferation in MCF-7 breast cancer cells via P2-purinoceptors. Br. J. Cancer 75, 34-39.

Dorsey, F.C., Steeves, M.A., Prater, S.M., Schroter, T., and Cleveland, J.L. (2009). Monitoring the autophagy pathway in cancer. Methods Enzymol. 453, 251-271.

Egan, D., Kim, J., Shaw, R.J., and Guan, K.L. (2011). The autophagy initiating kinase ULK1 is regulated via opposing phosphorylation by AMPK and mTOR. Autophagy 7, 643-644.

Eskelinen, E.L. (2008). New insights into the mechanisms of macroautophagy in mammalian cells. Int. Rev. Cell Mol. Biol. 266, 207-247.

Fabian, C.J., and Kimler, B.F. (2005). Selective estrogen-receptor modulators for primary prevention of breast cancer. J. Clin. Oncol. 23, 1644-1655.

Fisher, B., Costantino, J.P., Wickerham, D.L., Redmond, C.K., Kavanah, M., Cronin, W.M., Vogel, V., Robidoux, A., Dimitrov, N., Atkins, J., et al. (1998). Tamoxifen for prevention of breast cancer: report of the national surgical adjuvant breast and bowel project P-1 study. J. Natl. Cancer Inst. 90, 1371-1388.

Gizzo, S., Saccardi, C., Patrelli, T.S., Berretta, R., Capobianco, G., Di Gangi, S., Vacilotto, A., Bertocco, A., Noventa, M., Ancona, E., et al. (2013). Update on raloxifene: mechanism of action, clinical efficacy, adverse effects, and contraindications. Obstet. Gynecol. Surv. 68, 467-481.

$\mathrm{He}, \mathrm{C}$. , and Klionsky, D.J. (2009). Regulation mechanisms and signaling pathways of autophagy. Annu. Rev. Genet. 43, 67-93.
Hippert, M.M., O'Toole, P.S., and Thorburn, A. (2006). Autophagy in cancer: good, bad, or both? Cancer Res. 66, 9349-9351.

Hopfner, M., Lemmer, K., Jansen, A., Hanski, C., Riecken, E.O., Gavish, M., Mann, B., Buhr, H., Glassmeier, G., and Scherubl, H. (1998). Expression of functional P2-purinergic receptors in primary cultures of human colorectal carcinoma cells. Biochem. Biophys. Res. Commun. 251, 811-817.

Hwang, J.J., Kim, H.N., Kim, J., Cho, D.H., Kim, M.J., Kim, Y.S., Kim, Y., Park, S.J., and Koh, J.Y. (2010). Zinc(II) ion mediates tamoxifen-induced autophagy and cell death in MCF-7 breast cancer cell line. Biometals 23, 997-1013.

Jung, C.H., Ro, S.H., Cao, J., Otto, N.M., and Kim, D.H. (2010). mTOR regulation of autophagy. FEBS Lett. 584, 1287-1295.

Kanzawa, T., Zhang, L., Xiao, L., Germano, I.M., Kondo, Y., and Kondo, S. (2005). Arsenic trioxide induces autophagic cell death in malignant glioma cells by upregulation of mitochondrial cell death protein BNIP3. Oncogene 24, 980-991.

Khan, J.A., Forouhar, F., Tao, X., and Tong, L. (2007). Nicotinamide adenine dinucleotide metabolism as an attractive target for drug discovery. Expert Opin. Ther. Targets 11, 695-705.

Kim, J., Kundu, M., Viollet, B., and Guan, K.L. (2011). AMPK and mTOR regulate autophagy through direct phosphorylation of Ulk1. Nat. Cell Biol. 13, 132-141.

Lee, J.W., Park, S., Takahashi, Y., and Wang, H.G. (2010). The association of AMPK with ULK1 regulates autophagy. PLoS One 5, e15394.

Levine, B. (2007). Cell biology: autophagy and cancer. Nature 446 , 745-747.

Levine, B., and Kroemer, G. (2008). Autophagy in the pathogenesis of disease. Cell 132, 27-42.

Liang, X.H., Jackson, S., Seaman, M., Brown, K., Kempkes, B., Hibshoosh, H., and Levine, B. (1999). Induction of autophagy and inhibition of tumorigenesis by beclin 1 . Nature 402, 672-676.

Liu, Y.L., Yang, P.M., Shun, C.T., Wu, M.S., Weng, J.R., and Chen, C.C. (2010). Autophagy potentiates the anti-cancer effects of the histone deacetylase inhibitors in hepatocellular carcinoma. Autophagy 6, 1057-1065.

Maiuri, M.C., Zalckvar, E., Kimchi, A., and Kroemer, G. (2007). Selfeating and self-killing: crosstalk between autophagy and apoptosis. Nat. Rev. Mol. Cell Biol. 8, 741-752.

Mizushima, N., Yoshimori, T., and Levine, B. (2010). Methods in mammalian autophagy research. Cell 140, 313-326.

Morselli, E., Galluzzi, L., Kepp, O., Vicencio, J.M., Criollo, A., Maiuri, M.C., and Kroemer, G. (2009). Anti- and pro-tumor functions of autophagy. Biochim. Biophys. Acta 1793, 1524-1532.

Olivier, S., Close, P., Castermans, E., de Leval, L., Tabruyn, S., Chariot, A., Malaise, M., Merville, M.P., Bours, V., and Franchimont, N. (2006). Raloxifene-induced myeloma cell apoptosis: a study of nuclear factor-kappaB inhibition and gene expression signature. Mol. Pharmacol. 69, 1615-1623.

Powles, T. (2011). Prevention of breast cancer by newer SERMs in the future. Recent Results Cancer Res. 188, 141-145.

Rossi, V., Bellastella, G., De Rosa, C., Abbondanza, C., Visconti, D. Maione, L., Chieffi, P., Della Ragione, F., Prezioso, D., De Bellis, A., et al. (2011). Raloxifene induces cell death and inhibits proliferation through multiple signaling pathways in prostate cancer cells expressing different levels of estrogen receptor alpha and beta. J. Cell. Physiol. 226, 1334-1339.

Ryter, S.W., Cloonan, S.M., and Choi, A.M. (2013). Autophagy: a critical regulator of cellular metabolism and homeostasis. Mol. Cells 36, 7-16.

Shibata, M.A., Morimoto, J., Shibata, E., Kurose, H., Akamatsu, K. Li, Z.L., Kusakabe, M., Ohmichi, M., and Otsuki, Y. (2010). Raloxifene inhibits tumor growth and lymph node metastasis in a xenograft model of metastatic mammary cancer. BMC Cancer $10,566$.

Takeuchi, H., Kondo, Y., Fujiwara, K., Kanzawa, T., Aoki, H., Mills G.B., and Kondo, S. (2005). Synergistic augmentation of rapamycin-induced autophagy in malignant glioma cells by phosphatidylinositol 3-kinase/protein kinase B inhibitors. Cancer Res. 65 , 3336-3346.

Taurin, S., Allen, K.M., Scandlyn, M.J., and Rosengren, R.J. (2013). Raloxifene reduces triple-negative breast cancer tumor growth and decreases EGFR expression. Int. J. Oncol. 43, 785-792.

Wagstaff, S.C., Bowler, W.B., Gallagher, J.A., and Hipskind, R.A. 
Raloxifene Induces Autophagy via AMPK Activation Dong Eun Kim et al.

(2000). Extracellular ATP activates multiple signalling pathways and potentiates growth factor-induced $\mathrm{c}$-fos gene expression in MCF-7 breast cancer cells. Carcinogenesis 21, 2175-2181.

Wang, Q., Wang, L., Feng, Y.H., Li, X., Zeng, R., and Gorodeski, G.I. (2004). P2X7 receptor-mediated apoptosis of human cervical epithelial cells. Am. J. Physiol. Cell Physiol. 287, C1349-
1358.

White, N., and Burnstock, G. (2006). P2 receptors and cancer. Trends Pharmacol. Sci. 27, 211-217.

Yang, Z., and Klionsky, D.J. (2010). Mammalian autophagy: core molecular machinery and signaling regulation. Curr. Opin. Cell Biol. 22, 124-131. 